\title{
Irradiation combined with surgery for function preservation in the treatment of extramedullary plasmacytoma of the left labia majora during pregnancy: A case report
}

\author{
JINLAN HE ${ }^{1}$, YULONG ZHANG ${ }^{1}$, QIN SHEN ${ }^{1}$, YAN LI ${ }^{1}$, ZHE LIU ${ }^{1}$, \\ XINGCHEN PENG ${ }^{1}$, YALI SHEN ${ }^{1}$, RUI YIN ${ }^{2}$ and NIANYONG CHEN ${ }^{1}$ \\ ${ }^{1}$ Department of Radiation Oncology, Cancer Center and State Key Laboratory of Biotherapy; \\ ${ }^{2}$ Department of Pathology, West China Hospital, Sichuan University, Chengdu, Sichuan 610041, P.R. China
}

Received October 11, 2014; Accepted July 28, 2015

DOI: $10.3892 / \mathrm{ol} .2015 .3648$

\begin{abstract}
Extramedullary plasmacytoma (EMP) in the vulva is extremely rare. The current study presents, for the first time, a case of EMP in the left labia majora in a 36-year-old patient during pregnancy. A painful $7 \times 4 \times 2-\mathrm{cm}$ mass with ulceration, pus and blood scabs, previously misdiagnosed as vulvar ulcer in a local hosipital, was proven to be an EMP by biopsy. Upon magnetic resonance imaging, the tumor was shown to occur in the left labia majora without lymphadenopathy. A complete multiple myeloma (MM) workup excluded coexistence with MM. The goal of treatment was to eradicate the tumor while synchronously preserving the vulva. Therefore, following the termination of the pregnancy, radiotherapy with a total dose of 4,500 cGy markedly reduced the size of the tumor. An extended local excision of the residual tumor, and anaplasty of the vulva preserved the appearance and function of the vulva to the utmost. No post-operative radiation was administered, as the resection margins were not microscopically involved. There was no relapse, metastasis or progression to MM in a 9-month post-operative follow-up period, but close follow-up and long-term surveillance are required.
\end{abstract}

\section{Introduction}

Solitary plasmacytoma (SP) is a rare plasma cell dyscrasia that is characterized by the presence of bone or extramedullary monoclonal plasma cell tumors, without evidence of multiple myeloma (MM) (1). According to the location, SP can be categorized into two groups: Solitary plasmacytoma of the bone

Correspondence to: Professor Nianyong Chen, Department of Radiation Oncology, Cancer Center and State Key Laboratory of Biotherapy, West China Hospital, Sichuan University, 37 Guoxuexiang, Wuhou, Chengdu, Sichuan 610041, P.R. China E-mail: nchenyy@gmail.com

Key words: extramedullary plasmacytoma, labia majora, pregnancy, functional preservation
(SPB) and extramedullary plasmacytoma (EMP) (1). EMP accounts for $3-5 \%$ of all plasma cell tumors, with an incidence of only 0.04 cases per 100,000 individuals (2). Approximately $80 \%$ of EMPs occur in the upper respiratory tract (3), but they can also occur in numerous other sites $(3,4)$, including the gastrointestinal tract $(5,6)$, brain $(7)$, orbits $(8)$, thyroid gland (9), breasts (10), lungs (11), pleura (12), kidneys (13),bladder (14), urethra (15), ovaries (16), testes (17) and skin (18). Usually, EMPs have no specific clinical manifestations. They typically present as well-localized submucosal masses or swellings in the fifth to seventh decades of the life, with a male to female ratio of 3:1 (3). As the majority of patients can be cured by local radiotherapy, EMP carries an optimistic prognosis, with only $5-20 \%$ recurrence $(3,4,5,19)$.

EMP in the vulva is extremely rare (20) and to the best of our knowledge, no case involving the labia majora has previously been reported. The present study describes the first case of EMP occurring in the left labia majora in a young female during early pregnancy. The strengths of the case were the uncommon location and the function preservation treatment strategy. Written informed consent was obtained from the patient for inclusion in the present study.

\section{Case report}

In November 2013, a 36-year-old female (gravida 2, para 1) in the seventh week of pregnancy presented to the West China Hospital (Chengdu, China) with a gradually enlarging mass on the left labia majora, accompanied by ulceration and pain that had persisted for 2 months since August 2013. The mass presented as a small nodule $\sim 2 \mathrm{~cm}$ in diameter at the onset, with a rough surface and tenderness, which quickly expanded to the whole left labia majora and ulcerated with a purulent exudate. The diagnosis of a vulvar ulcer was made previously in a local hospital in October 2013, therefore, anti-inflammation treatment with intravenous benzylpenicillin ( 3 million units every $8 \mathrm{~h}$ for 10 consecutive days) and debridement were performed. However, the symptoms were not alleviated and the patient was subsequently transferred to the West China Hospital.

Upon admission to the West China Hospital in November 2013, an irregular 7x4×2-cm mass was found in the 

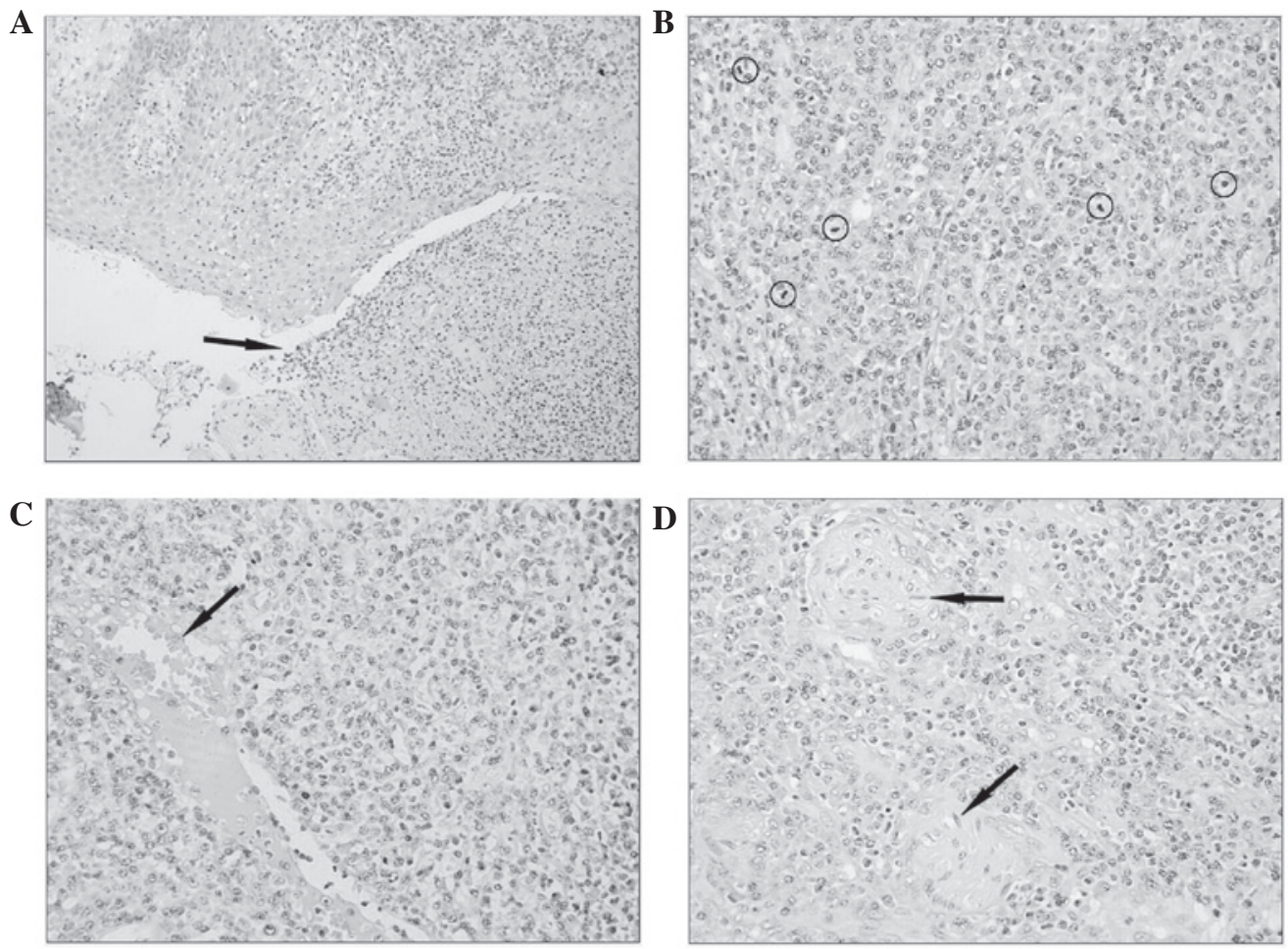

Figure 1. Histological analyses of the specimen (hematoxylin and eosin staining). (A) Skin ulcer (black arrow), and diffuse dermal and subcutaneous infiltration with plasmacytoid cells (magnification, x200). (B) Plasmacytoid cells at mitotic phase (black circles) (magnification, x400). (C) Plasmacytoid cells invading the blood vessel (black arrow) (magnification, x400). (D) Plasmacytoid cells invading the nerves (black arrows) (magnification, $\mathrm{x} 400$ ).
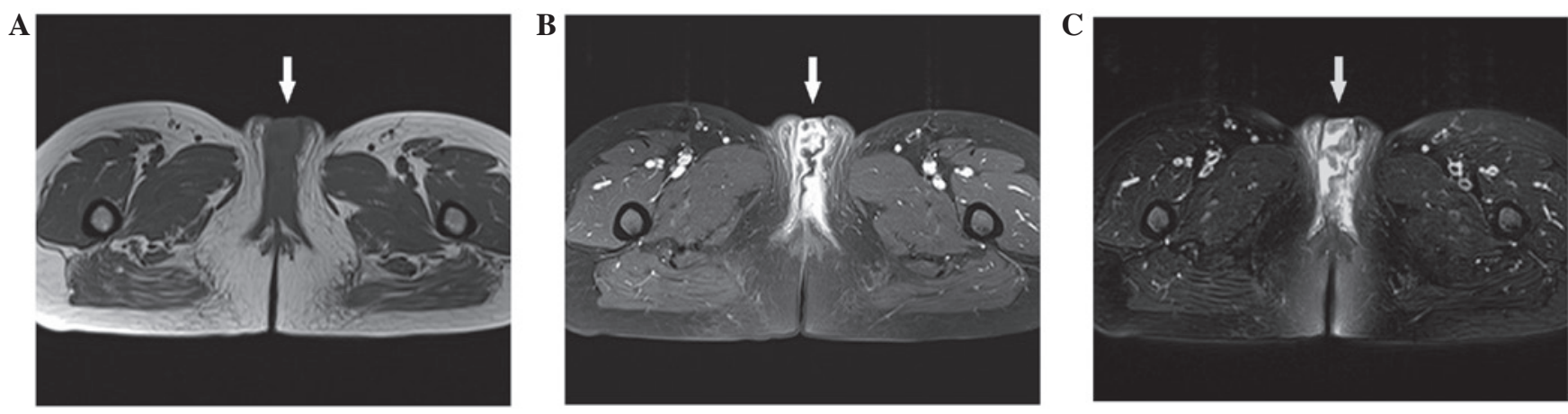

Figure 2. Magnetic resonance imaging scans revealing that the mass (white arrows) was located in the vulva and measured $7.1 \times 3.4 \mathrm{~cm}$. No inguinal lymph node enlargement was apparent. (A) T1-weighted plain scan. (B) T1-weighted enhanced scan. (C) T2-weighted scan.

left labia majora, with blood scabs and a purulent exudate. The mass was hard and tender on palpation. There were no palpable inguinal lymph nodes. The patient reported persistent spontaneous pain with a score of 6 in the numerical rating scale (21).

A biopsy was performed and the histopathology results revealed a diffuse infiltration of plasmacytoid cells in the dermis and subcutaneous tissue, a number of which invaded the nerves and vessels (Fig. 1). The cells were positive for cluster of differentiation (CD)79a and $\kappa$-light chain, partly positive for CD132, and negative for CD20, $\lambda$-light chain, CD23, CD5, CD3, B-cell lymphoma (Bcl)-2, Bcl-6 and CD10. Ki-67 staining revealed a high cell proliferation rate with $\sim 50 \%$ immunoreactive cells. No monoclonal gene rearrangement of immunoglobulin $(\mathrm{Ig}) \mathrm{H}$ or IgK was detected. Therefore, a diagnosis of EMP was proposed.

Subsequently, an extensive medical workup was performed to delineate the extent of the lesion and to rule out coexistence with MM. As the patient had decided to terminate the pregnancy, imaging examinations were conducted. An irregular mass with mixed signals and without lymphadenopathy was found on magnetic resonance imaging (MRI) (Fig. 2). No metastases were found on chest and abdominal computed tomography (CT) scans. A normal result with only $0.5 \%$ plasma cells was yielded upon bone marrow aspiration and biopsy. No bone lesions were found on bone single-photon emission CT. No anemia, hypercalcemia or renal impairment were detected. Serum electrophoresis did not show the M-band and Bence-Jones proteinuria was not detected. Serum albumin and Ig levels were normal, and serum and urinary $\beta 2$ microglobulin levels were unremarkable. On the basis of these results, a definitive diagnosis of EMP was established.

Following termination of the pregnancy, pre-operative external irradiation with a 6-MV photon beam though an 

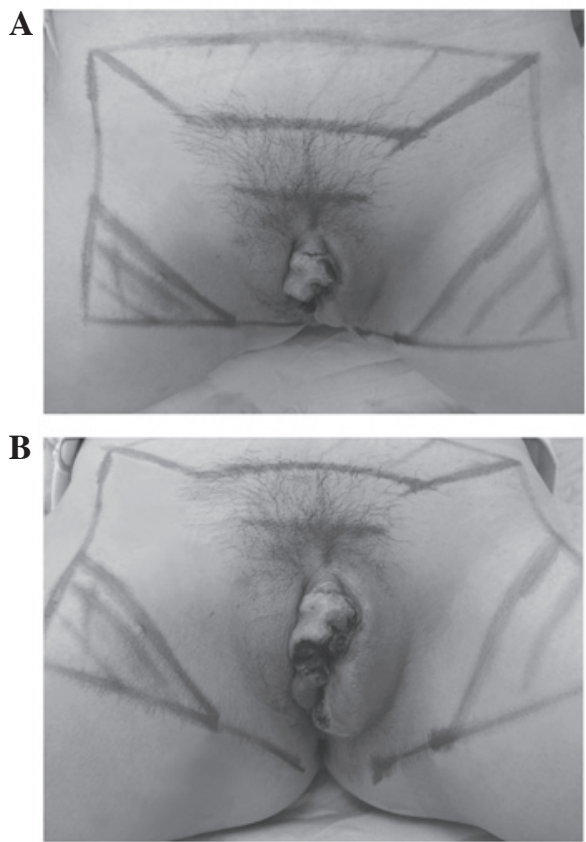

Figure 3. Radiotherapy plan. The patient was treated in the supine 'frog-leg' position, with knees apart and feet together. The radiation field was $22 \times 14 \mathrm{~cm}$ in size, with the upper, lower and bilateral boundaries reaching the pubic symphysis, the posterior border of the labia majora and the inside two-thirds of the inguinal regions, respectively. Diagonal lines represented blocks. An isocentric, source skin distance technique was adopted and 6-MV X-rays measured at a depth of $3 \mathrm{~cm}$ were applied through an anterior portal. (A) Top view. (B) Front view.

anterior portal was applied to the vulvar area and the inguinofemoral lymph nodes (Fig. 3) using daily fractionation of 200 cGy in five fractions weekly (21 fractions in total), except for the third fraction in which 500 cGy was adopted due to aggravated pain. Dynamic observations of the mass were made every week. No severe moist desquamation or maceration of the perineal skin occured. The total 4,500-cGy treatment was completed over 32 days, and compared with the initial presentation (Fig. 4A), the tumor was satisfactorily reduced in size (Fig. 4B).

Subsequently, 40 days later, the mass had further decreased in size and only two small nodules existed (Fig. 4C). The mass was reevaluated and the surgical timing was appropriate. An extended local excision and anaplasty of the vulva was performed. As the post-operative pathology confirmed that no tumor cells were present, post-operative boost irradiation was not performed. The post-operative course was uneventful (Fig. 4D) and the patient was advised to attend regular follow-ups every 3 months. At 9 months post-surgery, there was no evidence of local recurrence, distant metastasis or progression to MM. The patient has continued to maintain a satisfactory sexual life.

\section{Discussion}

EMP of the labia majora is extremely rare. The current study presents, to the best of our knowledge, the first documented case of human EMP involving the labia majora and the first such case presenting during pregnancy. A thorough literature search in Pubmed found only one study reporting a case of simultaneous EMPs of the vagina and right labia minora, with a literature
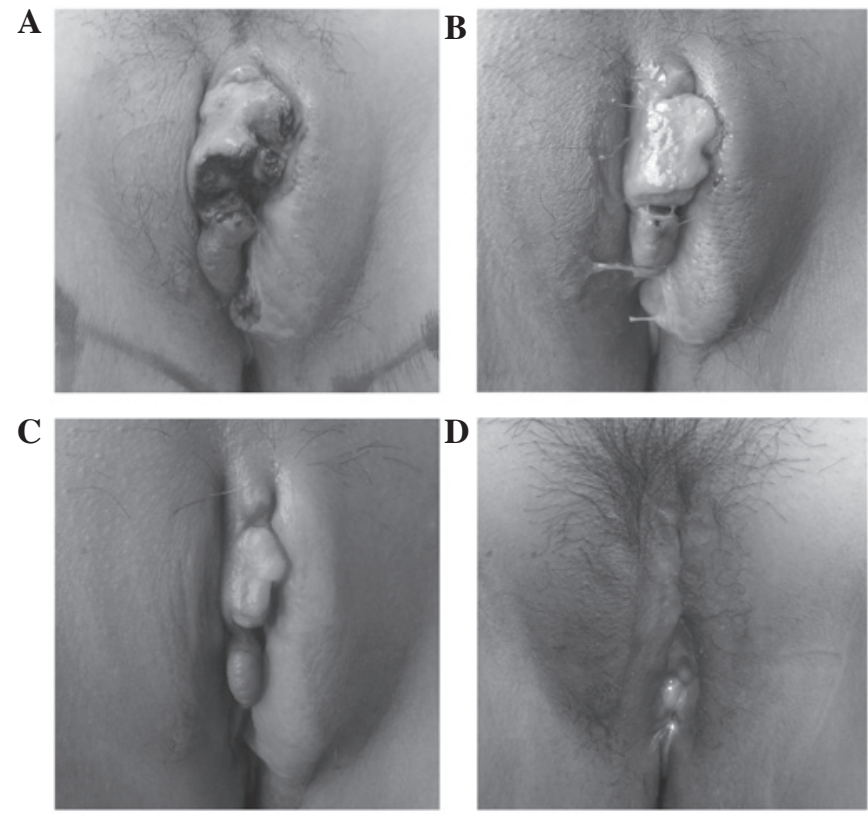

Figure 4. Vulvar appearances prior to, during and following treatment. (A) 3 days prior to radiotherapy, an irregular mass $\sim 7 \times 4 \times 2 \mathrm{~cm}$ in size was present in the swollen left labia majora, with blood scabs and a purulent exudate. (B) 1 day after radiotherapy, the mass had been reduced in volume by two-thirds, but more exudate was present. (C) 41 days after radiotherapy and 2 days prior to surgery, the mass had been further reduced in size, and only two nodules, $\sim 2 \times 1 \times 1$ and $1 \times 0.5 \times 0.5 \mathrm{~cm}$ in size, remained. The acute edema had disappeared. (D) 2 months after surgery, the vulvar appearance had returned basically to normal.

review of four cases invading the vagina and with a mention of three vulvar plasmacytomas (20). However, the present case is reported not only for its uncommon location, but also for its tortuous diagnosis, treatment strategy and close follow-up.

The differential diagnosis should be kept in mind and rare diseases should be considered when diagnosing all masses. Due to the rare occurance and variety of clinical manifestations of EMP, an accurate diagnosis is often delayed. In the present case, the patient was initially misdiagnosed with a vulvar ulcer in a local hospital. In fact, the main feature of the disease was the progressively enlarging mass, while the ulceration was only an accompanying symptom. When treatment effectiveness cannot be achieved, rare diseases should be considered. Pathological biopsy, a golden criteria of diagnosis, is essential for improving diagnostic accuracy.

An EMP was proposed on the basis of diffuse monoclonal plasma cell infiltration in the biopsy tissue of the present patient. Once EMP is proposed by pathology, a medical workup should be arranged to exclude existence of MM. The recommended diagnostic criteria for EMP are: i) A single extramedullary mass of clonal plasma cells; ii) histologically normal bone marrow aspirate and trephine; iii) normal results on skeletal survey, including radiology of the long bones; iv) no anemia, hypercalcaemia or renal impairment due to plasma cell dyscrasia; and v) an absent or low serum or urinary level of monoclonal Ig (22). In the present case, all relevant tests had been conducted to exclude coexistence with MM.

Due to the small number of patients and lack of randomized controlled trials, there are no established criteria for the treatment of EMP. However, radiotherapy is considered as 
the mainstay due to the high radiosensitivity of EMP, particularly in the head and neck area where resection is limited by the anatomical complexity. Several series have reported $80-100 \%$ local control rates $(4,5,19,23-27)$. The optimal radiation dose recommended by the United Kingdom Myeloma Forum is in the range of 40-50 Gy $(22,25)$. A dose of $40 \mathrm{~Gy}$ in 20 fractions can confer an excellent chance of local control in tumors that are $\leq 5 \mathrm{~cm}$ in diameter, whereas a higher dose of $\sim 50 \mathrm{~Gy}$ in 25 fractions is required in tumors $>5 \mathrm{~cm}$ due to a higher risk of local failure $(27,28)$. The necessity of prophylactic irradiation of local lymph nodes is unclear, as excellent results could be achieved with elective inclusion of the draining lymph nodes (26) and inclusion of them only when clinically involved $(27,29)$. Complete surgical excision can be considered at other sites outside the head and neck area if feasible. A combination of radiotherapy with surgery has been demonstrated to be less invasive and to produce better overall survival (30). The role of adjuvant chemotherapy is inconclusive and additional use of it may bring certain benefits to patients with large masses and high-grade histology (22).

For the present patient, radiotherapy dominated the treatment not only due to its effectiveness, but also due to the advantage of function preservation. If surgery had been adopted first, the destruction of the vulvar appearance or even function may have caused major psychosexual problems in this patient of reproductive age. A moderate dose of 4,500 cGy was considered enough due to its predetermined combination with the subsequent radical surgery. The inguinal lymph node region was prophylactically irradiated for safety. It is noteworthy that the temporary adjustment to $500 \mathrm{cGy}$ irradiation for the third fraction contributed greatly to the pain relief without injury to the urethra. The external location of the tumor made direct observation, without the requirement for CT or MRI, easy. Therefore, the change in tumor size and appearance demonstrated vividly the radiosensitivity of EMP, which has been rarely reported in other cases. Surgical timing was determined to be appropriate $\sim 1$ month after radiotherapy to avoid acute edema immediately after radiotherapy and to make best use of the late effect of radiotherapy. Overall, as indicated in the present case, the primary treatment for the majority of patients will be radiotherapy, but surgery may also be required. Close communication between the hematologist, radiotherapist and surgeon is therefore vital for providing optimum care.

EMP has the best prognosis of all plasma cell tumors. The 5-year overall survival rate varies between 31 and $82 \%(5,25,29,31,32)$. However, unlike other tumors in which attention is focused more on relapse and distant metastasis during follow-up, EMP requires extra attention with regard to progression to MM, as the majority of mortalities associated with EMP are due to this conversion. Compared with SPB, progression of EMP to MM occurs less frequently. Approximately $60 \%$ of patients with SPB develop MM $(27,28,33)$ while EMP progresses to MM in 5-44\% of cases $(3,5,23,28,29,33,34)$. Although progression to MM usually occurs within 2 years of the initial diagnosis, conversion has occurred up to 15 years later $(3,5,19,23,30)$. Therefore, the long-term regular evaluation of patients with EMP is strongly recommended. A physical examination, imaging of the primary site, bone marrow aspiration and biopsy, radiographic studies of the skeleton, serum protein electrophoresis, free light chain assays and laboratory tests, including a complete blood count, renal function tests, and analyses of blood calcium, serum albumin and Ig levels, are required during follow-ups.

In conclusion, the present study, for the first time, reports a case of EMP involving the left labia majora in a pregnant woman. The combination of irradiation and surgery leads to excellent tumor control and function preservation. Regular follow-ups should be performed in such patients in case of relapse, metastasis or progression to $\mathrm{MM}$.

\section{References}

1. Galieni P, Cavo M, Avvisati G, Pulsoni A, Falbo R, Bonelli MA, Russo D, Petrucci MT, Bucalossi A and Tura S: Solitary plasmacytoma of bone and extramedullary plasmacytoma: Two different entities? Ann Oncol 6: 687-691, 1995.

2. Perez CA: Unusual nonepithelial tumors of the head and neck In: Principles and Practice of Radiation Oncology. Perez CA and Brady LW (eds). 3rd edition. Lippincott Raven Publishers, Philadelphia, pp1116-1117, 1997.

3. Alexiou C, Kau RJ, Dietzfelbinger H, Kremer M, Spiess JC, Schratzenstaller B and Arnold W: Extramedullary plasmacytoma: Tumor occurrence and therapeutic concepts. Cancer 85: 2305-2314, 1999.

4. Chao MW, Gibbs P, Wirth A, Quong G, Guiney MJ and Liew KH: Radiotherapy in the management of solitary extramedullary plasmacytoma. Intern Med J 35: 211-215, 2005.

5. Liebross RH, Ha CS, Cox JD, Weber D, Delasalle K and Alexanian R: Clinical course of solitary extramedullary plasmacytoma. Radiother Oncol 52: 245-249, 1999.

6. Galieni P, Cavo M, Pulsoni A, Avvisati G, Bigazzi C, Neri S, Caliceti U, Benni M, Ronconi S and Lauria F: Clinical outcome of extramedullary plasmacytoma. Haematologica 85: 47-51, 2000.

7. Manabe M, Kanashima H, Yoshii Y,Mukai S, Sakamoto E, Iwai Y, Kubo Y, Fukushima H, Inoue $\mathrm{T}$ and Teshima H: Extramedullary plasmacytoma of the dura mimicking meningioma. Int $\mathrm{J}$ Hematol 91: 731-732, 2010.

8. Duletic-Nacinovic A, Stifter S, Marijic B, Miletic D, Loncarek K, Manestar D and Jonjic N: Dacryocystitis provoked by recurrence of extramedullary plasmacytoma of the orbit: A case report. Tumori 96: 164-167, 2010.

9. Puliga G, Olla L, Bellisano G, Di Naro N, Ganau M, Lai ML, Faa $\mathrm{G}$ and Tolu GA: Solitary extramedullary plasmacytoma of the thyroid gland associated with multinodular goiter: Case report and review of the literature. Pathologica 103: 61-63, 2011.

10. De Chiara A, Losito S, Terracciano L, Di Giacomo R, Iaccarino G and Rubolotta MR: Primary plasmacytoma of the breast. Arch Pathol Lab Med 125: 1078-1080, 2001.

11. Ujiie H, Okada D, Nakajima Y, Yoshino N and Akiyama H: A case of primary solitary pulmonary plasmacytoma. Ann Thorac Cardiovasc Surg 18: 239-242, 2012.

12. Feng PH, Huang CC, Wang CW, Wu YK and Tsai YH: Solitary pleural plasmacytomas manifested as a massive pleural effusion without evidence of monoclonal gammopathy. Respirology 13: 751-753, 2008.

13. Zhang SQ, Dong P, Zhang ZL, Wu S, Guo SJ, Yao K, Li YH, Liu ZW, Han H, Qin ZK, et al: Renal plasmacytoma: Report of a rare case and review of the literature. Oncol Lett 5: 1839-1843, 2013.

14. Khaliq W, Uzoaru I, Konchanin RP, Sapiente RA and Egner JR: Solitary extramedullary plasmacytoma of the bladder: A case report and literature. Oncology (Williston Park) 24: 832-835, 2010.

15. Klein T, Holz A, Neid M, Hinkel A and Noldus J: The first description of an extramedullary plasmacytoma of the ureter. Urol Int 84: 122-124, 2010.

16. Emery JD, Kennedy AW, Tubbs RR, Castellani WJ and Hussein MA: Plasmacytoma of the ovary: A case report and literature review. Gynecol Oncol 73: 151-154, 1999.

17. Iizumi T, Shinohara S, Amemiya H, Tomomasa H, Yazaki T, Umeda T, Tanaka F and Imamura T: Plasmacytoma of the testis. Urol Int 55: 218-221, 1995. 
18. Koletsa T, Patsatsi A, Kostopoulos I, Kartsios C, Korantzis I and Sotiriadis D: A case of a primary cutaneous plasmacytoma presenting in adolescence. Am J Dermatopathol 34: 537-540, 2012.

19. Bachar G, Goldstein D, Brown D, Tsang R, Lockwood G, Perez-Ordonez B and Irish J: Solitary extramedullary plasmacytoma of the head and neck-long-term outcome analysis of 68 cases. Head Neck 30: 1012-1019, 2008.

20. Doss LL: Simultaneous extramedullary plasmacytomas of the vagina and vulva: A case report and review of the literature. Cancer 41: 2468-2474, 1978.

21. Breivik H, Borchgrevink PC, Allen SM, Rosseland LA, Romundstad L, Hals EK, Kvarstein G and Stubhaug A: Assessment of pain. Br J Anaesth 101: 17-24, 2008.

22. Soutar R, Lucraft H, Jackson G, Reece A, Bird J, Low E and Samson D; Guidelines Working Group of the UK Myeloma Forum; British Committee for Standards in Haematology; British Society for Haematology: Guidelines on the diagnosis and management of solitary plasmacytoma of bone and solitary extramedullary plasmacytoma. Br J Haematol 124: 717-726, 2004.

23. Michalaki VJ, Hall J, Henk JM, Nutting CM and Harrington KJ: Definitive radiotherapy for extramedullary plasmacytomas of the head and neck. Br J Radiol 76: 738-741, 2003.

24. Creach KM, Foote RL, Neben-Wittich MA and Kyle RA: Radiotherapy for extramedullary plasmacytoma of the head and neck. Int J Radiat Oncol Biol Phys 73: 789-794, 2009.

25. Tournier-Rangeard L, Lapeyre M, Graff-Caillaud P, Mege A, Dolivet G, Toussaint B, Charra-Brunaud C, Hoffstetter S, Marchal C and Peiffert D: Radiotherapy for solitary extramedullary plasmacytoma in the head-and-neck region: A dose greater than $45 \mathrm{~Gy}$ to the target volume improves the local control. Int J Radiat Oncol Biol Phys 64: 1013-1017, 2006.

26. Bolek TW, Marcus RB Jr and Mendenhall NP: Solitary plasmacytoma of bone and soft tissue. Int J Radiat Oncol Biol Phys 36 329-333, 1996.
27. Tsang RW, Gospodarowicz MK, Pintilie M, Bezjak A, Wells W, Hodgson DC and Stewart AK: Solitary plasmacytoma treated with radiotherapy: Impact of tumor size on outcome. Int J Radiat Oncol Biol Phys 50: 113-120, 2001.

28. Holland J, Trenkner DA, Wasserman TH and Fineberg B: Plasmacytoma. Treatment results and conversion to myeloma. Cancer 69: 1513-1517, 1992.

29. Susnerwala SS, Shanks JH, Banerjee SS, Scarffe JH, Farrington WT and Slevin NJ: Extramedullary plasmacytoma of the head and neck region: Clinicopathological correlation in 25 cases. Br J Cancer 75: 921-927, 1997.

30. Sasaki R, Yasuda K, Abe E, Uchida N, Kawashima M, Uno T, Fujiwara M, Shioyama Y, Kagami Y, Shibamoto Y, et al: Multi-institutional analysis of solitary extramedullary plasmacytoma of the head and neck treated with curative radiotherapy. Int J Radiat Oncol Biol Phys 82: 626-634, 2012.

31. Kapadia SB, Desai U and Cheng VS: Extramedullary plasmacytoma of the head and neck. A clinicopathologic study of 20 cases. Medicine (Baltimore) 61: 317-329, 1982.

32. Miller FR, Lavertu P, Wanamaker JR, Bonafede J and Wood BG: Plasmacytomas of the head and neck. Otolaryngol Head Neck Surg 119: 614-618, 1998.

33. Mayr NA, Wen BC, Hussey DH, Burns CP, Staples JJ, Doornbos JF and Vigliotti AP: The role of radiation therapy in the treatment of solitary plasmacytomas. Radiother Oncol 17: 293-303, 1990 .

34. Katodritou E, Terpos E, Symeonidis AS, Pouli A, Kelaidi C, Kyrtsonis MC, Kotsopoulou M, Delimpasi S, Christoforidou A, Giannakoulas N, et al: Clinical features, outcome and prognostic factors for survival and evolution to multiple myeloma of solitary plasmacytomas: A report of the Greek myeloma study group in 97 patients. Am J Hematol 89: 803-808, 2014. 